\title{
The Poisson-Sigma Model A Non-Linear Gauge Theory*
}

\author{
THOMAS SCHWARZWELLER ${ }^{\dagger}$ \\ Fachbereich Physik \\ Universität Dortmund
}

October 26, 2018

\begin{abstract}
I investigate the PoIsson-sigma model on the classical and quantum level. First I show how the interaction can be obtained by a deformation of the classical master equation of an Abelian BF theory in two dimensions. On the classical level this model includes various known two-dimensional field theories, in particular the YANG-MiLls theory. On the quantum level the perturbation expansion of the path integral in the covariant gauge yields the KONTSEVICH deformation formula. Finally I perform the calculation of the path integral in a general gauge, and demonstrate how the derived partition function reduces in the special case of a linear PoIsSON structure to the familiar form of 2d YANG-MiLLS theory.
\end{abstract}

\section{Introduction}

The class of non-linear gauge theories introduced by IKEDA in [9] is based on a polynomial extension of the underlying LIE algebra, for instance a finite $\mathrm{W}$-algebra or a POISSON algebra. Due to this non-linearity these models involve in the language of gauge theories an open gauge algebra, i.e. the algebra closes only on-shell. In such cases neither the FADDEEVPOPOV quantization nor the BRST procedure leads to an appropriate application of a path integral quantization since both needs a well-defined cohomology to construct physical variables but the corresponding BRST operator is only nilpotent modulo the equations of motion. The proper method that works in these cases is the BATALIN-VILKOVISKY procedure, for a detailed description see [6]. This formalism has a beautiful geometric interpretation that enables one to receive the extended action used in the path integral from fundamental geometric ingredients, a nilpotent vector field and a symplectic structure on appropriate super manifolds [1]. A famous representative of a non-linear gauge theory is the PoIsson-sigma model 13. which obviously is a sigma model with PoIsson structure on the target manifold. The interest in the last years is essentially based on its connection to the KONTSEVICH formula for deformation quantization of Poisson manifolds [12] discovered by CATTANEO and Felder 洎]. Another important aspect is that the classical model associates to certain Poisson structures on a finite-dimensional manifold two-dimensional field theories. For a linear PoIssON

*Talk given at the 3rd International Conference on Geometry, Integrability and Quantization in Varna, Bulgaria, June 14-23 2001

† thomas@doom.physik. uni-dortmund.de 
structure which leads to the two-dimensional YANG-MiLLS theory it was possible to show this connection also on the quantum level, i.e. the partition function of both theories corresponds to each other [7].

Also based on the BATALIN-VILKOVISKY procedure, strictly speaking on the classical master equation, is a method to generate a physical consistent interaction between the gauge fields of a free gauge theory [2]. This problem then turns into a deformation of the solution of the master equation which is possible since it contains all the information about the considered gauge structure.

This article is structured as follows. In section 2 the classical theory of the PoIsson-sigma model will be presented. That includes a short review of the general method to construct an interaction and the derivation of the model by means of a deformation of a free BF theory. In section 3 then the quantization will be performed, in particular the FEYNMAN perturbation series [4] and the non perturbative calculation of the partition function [7].

\section{The Classical Theory}

\subsection{Consistent Interaction via Deformation}

In this section the cohomological approach to the problem of generating consisting interactions is reviewed [2]. It essentially is based on the antifield formalism in the sense that a deformation of the solution of the master equation leads to an action functional containing a consistent interaction term.

First of all I present a very brief review of the antifield formalism. Indeed, here are just some properties which will be needed in the forthcoming sections. The starting point is an action $S_{0}[\phi]$ with gauge symmetries $\delta_{\epsilon} \phi^{i}=\mathcal{R}_{\alpha}^{i} \epsilon^{\alpha}$. By introducing ghosts and antifields one can construct a solution $S\left[\phi^{A}, \phi_{A}^{*}\right]$ of the master equation

$$
(S, S)=\frac{\delta S}{\delta \phi^{A}} \frac{\delta S}{\delta \phi_{A}^{*}}-\frac{\delta S}{\delta \phi_{A}^{*}} \frac{\delta S}{\delta \phi^{A}}=0, \quad \text { with } S=S_{0}+\phi_{i}^{*} \mathcal{R}_{\alpha}^{i} C^{\alpha}+\cdots
$$

where $\phi^{A}=\left(\phi^{i}, C^{\alpha}, \ldots\right)$ denotes the sets of the original fields, the ghosts and so on, while $\phi_{A}^{*}$ stands for the corresponding antifields. In the solution of the master equation the whole information about the gauge structure is encoded. The algebra of the fields and antifields together with the BRST-differential generated by $S$ through the antibracket

$$
s(\cdot)=(\cdot, S)
$$

yields a complex. The corresponding BRST cohomology is denoted by $H^{\star}=\sum H^{p}(s)$. One can define a map in the cohomology induced by the antibracket, the antibracket map

$$
\begin{gathered}
(\cdot, \cdot): H^{p}(s) \times H^{q}(s) \longrightarrow H^{(p+q+1)}(s), \\
([A],[B]) \mapsto[(A, B)] .
\end{gathered}
$$

The important result found by G.BARNICH and M.HEnNEAUX is the fact that the antibracket map is trivial in the sense the antibracket of 2 BRST-closed functionals is BRST-exact, for a proof consult [2]. Due to the triviality of the antibracket map one can define higher order maps in the cohomology, however it turns out they are trivial in a similar way. 
Now consider a free gauge theory with a free symmetry given by

$$
\text { Free Theory }=\left\{\begin{array}{l}
\text { Free action }: \stackrel{(0)}{S}{ }_{0}\left[\phi^{i}\right] \\
\text { Gauge Symmetry }: \delta_{\epsilon} \phi^{i}=\stackrel{(0)}{\mathcal{R}}_{\alpha}^{i} \epsilon^{\alpha} \\
\text { Noether Theorem }: \frac{\stackrel{(0)}{S}_{S_{0}\left[\phi^{i}\right]}}{\delta \phi^{i}} \stackrel{(0)}{\mathcal{R}}^{i}{ }_{\alpha}=0 .
\end{array}\right.
$$

The aim is now to introduce couplings between the fields $\phi^{i}$ which fulfill the crucial physical requirement of preserving the number of gauge symmetries, those couplings will be called consistent. It means one has to perturb the action and the symmetries

$$
\begin{gathered}
\stackrel{(0)}{S_{0}} \longrightarrow S_{0}=\stackrel{(0)}{S}_{0}+g \stackrel{(1)}{S}_{0}+g^{2} \stackrel{(2)}{S}_{0}+\ldots, \\
\stackrel{(0)}{\mathcal{R}}_{\alpha}{ }_{\alpha} \longrightarrow \mathcal{R}_{\alpha}^{i}=\stackrel{(0)}{\mathcal{R}}^{i}{ }_{\alpha}+g \stackrel{(1)}{\mathcal{R}}^{i}{ }_{\alpha}+g^{2} \stackrel{(2)}{\mathcal{R}}_{\alpha}^{i}+\ldots,
\end{gathered}
$$

such that $\delta_{\epsilon} \phi^{i}=\mathcal{R}_{\alpha}^{i} \epsilon^{\alpha}$ is a symmetry of $S_{0}$

$$
\frac{\delta\left(\stackrel{(0)}{S}_{0}+g \stackrel{(1)}{S}_{0}+g^{2} \stackrel{(2)}{S}_{0}+\ldots\right)}{\delta \phi^{i}}\left(\stackrel{(0)}{\mathcal{R}}_{\alpha}^{i}+g \stackrel{(1)}{\mathcal{R}}_{\alpha}^{i}+g^{2} \stackrel{(2)}{\mathcal{R}}_{\alpha}^{i}+\ldots\right)=0
$$

which expresses the consistency. It is not an easy task to deform simultaneously the action and the symmetry to get a consistent interaction.

This problem can be reformulated as a deformation problem of the solution of the master equation. Basically this procedure is based on the fact that the master equation contains all the information about the gauge structure

$$
\begin{gathered}
\left(\stackrel{(0)}{S}_{B V}, \stackrel{(0)}{S}_{B V}\right)=0 \longrightarrow\left(S_{B V}, S_{B V}\right)=0, \\
\stackrel{(0)}{S}_{B V} \longrightarrow S_{B V}=\stackrel{(0)}{S}_{B V}+g \stackrel{(1)}{S}_{B V}+g^{2} \stackrel{(2)}{S}_{B V}+\ldots .
\end{gathered}
$$

The deformed master equation guarantees now the consistency of $S_{0}$ and $\mathcal{R}_{\alpha}^{i}$ and further on the original and the deformed gauge theory have the same spectrum of ghosts and antifields.

The advantage of this formulation is that one now can use the cohomological techniques of deformation theory. The deformed master equation can be analyzed order by order in the deformation parameter, the coupling constant. This expansion yields the following relations

$$
\begin{gathered}
\left(\stackrel{(0)}{S}_{B V}, \stackrel{(0)}{S}_{B V}\right)=0, \\
2\left(\stackrel{(0)}{S}_{B V}, \stackrel{(1)}{S}_{B V}\right)=0, \\
2\left(\stackrel{(0)}{S}_{B V}, \stackrel{(2)}{S}_{B V}\right)+\left(\stackrel{(1)}{S}_{B V}, \stackrel{(1)}{S}_{B V}\right)=0, \\
(+ \text { higher orders }) .
\end{gathered}
$$


The first equation (2.10) is fulfilled by assumption, it is exactly the master equation for the free gauge theory. Equation (2.11) shows that $\stackrel{(1)}{S}_{B V}$ is forced to be a cocycle of the free BRST differential $\stackrel{(0)}{s}$. Assume now $\stackrel{(1)}{S}_{B V}$ is a coboundary then the corresponding interaction belongs to a field redefinition which should not be considered and the deformation will be called trivial. Therefore $\stackrel{(1)}{S}_{B V}$ is an element of the zeroth cohomological space $H^{0}(\stackrel{(0)}{s})$ which is isomorphic to the space of physical observables of the free theory. Because of the triviality of the antibracket map $\left(\stackrel{(1)}{S}_{B V}, \stackrel{(1)}{S}_{B V}\right)$ is BRST exact and one gets no obstructions for constructing the interaction from $(2.12)$ and $\stackrel{(2)}{S}_{B V}$ exist. This is also true for higher orders, so there are no obstructions for the interacting action at all.

Usually the original action is a local functional of a corresponding LAGRANGE function, such that also the deformations need to be local functionals. Taking locality into account the analysis gets much more involved because the antibracket map is not trivial anymore, e.g. the antibracket of two local BRST cocycles need not necessarily to be the BRST variation of a local functional. Consider $\stackrel{(k)}{S}=\int \stackrel{(k)}{\mathcal{L}}$, where $\mathcal{L}$ is the Lagrangian, an n-form. The corresponding (local) antibracket is defined modulo an d-exact term, $\mathrm{d}$ being the exterior derivative. This yields for the deformation expansion of the Lagrangian

$$
\begin{gathered}
2 \stackrel{(0)(1)}{S} \stackrel{\mathcal{L}}{=} \mathrm{d} \stackrel{(1)}{j}, \\
\stackrel{(0)(2)}{S} \mathcal{L}+\{\stackrel{(1)}{\mathcal{L}}, \stackrel{(1)}{\mathcal{L}}\}=\mathrm{d} \stackrel{(2)}{j}, \\
(+ \text { higher orders }),
\end{gathered}
$$

where $\stackrel{(k)}{j}$ is the symbol for the d-exact term. $\stackrel{(1)}{\mathcal{L}}$ is BRST closed modulo d, this means that the nontrivial deformations of the master equations belong to $H^{0}(\stackrel{(0)}{s} \mid \mathrm{d})$. Because the corresponding local antibracket is no longer trivial, it possesses a lot of structure, one gets obstructions for the construction of the interaction term, some so-called consistency conditions. The construction of local consistent interaction is strongly constrained.

\subsection{Deformation of Abelian BF Theory}

As an application of the formalism consider now the nontrivial deformation of Abelian BF theory in two dimensions [10]. The free action is given by

$$
\stackrel{(0)}{S}_{0}=\int_{\Sigma_{g}} A_{i} \wedge \mathrm{d} \phi^{i},
$$

which is invariant under the gauge transformation

$$
\delta \phi^{i}=0, \quad \delta A_{i}=\mathrm{d} \epsilon_{i} .
$$

The minimal solution of the classical master equation is

$$
\stackrel{(0)}{S}=\int_{\Sigma_{g}}\left[A_{i} \wedge \mathrm{d} \phi^{i}+A^{* i} \mathrm{~d} C_{i}\right]
$$


and the corresponding BRST-Symmetry is

$$
\stackrel{(0)}{s}=\mathrm{d} C_{i} \frac{\vec{\partial}}{\partial A_{i}}+\mathrm{d} \phi^{i} \frac{\vec{\partial}}{\partial A^{* i}}+\mathrm{d} A_{i} \frac{\vec{\partial}}{\partial \phi_{i}^{*}}-\mathrm{d} A^{* i} \frac{\vec{\partial}}{\partial C^{* i}} .
$$

The first order deformation $\stackrel{(1)}{\mathcal{L}}$ of the Lagrangian associated to the minimal solution $\stackrel{(0)}{S}$ should obey the following condition

$$
\stackrel{(0)}{s} \stackrel{(1)}{\mathcal{L}}+\mathrm{d} a_{[1]}=0
$$

It defines an element of $H^{0}(\stackrel{(0)}{s} \mid \mathrm{d})$, so that one gets a set of descent equations

$$
\stackrel{(0)}{s} a_{[1]}+\mathrm{d} a_{[o]}=0, \stackrel{(0)}{s} a_{[0]}=0 .
$$

It is a simple calculation to get the solution for $\stackrel{(1)}{\mathcal{L}}$

$$
\begin{aligned}
\stackrel{(1)}{\mathcal{L}}=-\frac{1}{4} \frac{\delta^{2} f^{i j}[\phi]}{\delta \phi^{k} \delta \phi^{l}} A^{* k} \wedge A^{* l} C_{i} C_{j} & +\frac{\delta f^{i j}[\phi]}{\delta \phi^{k}} C^{* k} C_{i} C_{j} \\
& -\frac{\delta f^{i j}[\phi]}{\delta \phi^{k}} A^{* k} \wedge A_{i} C_{j}-f^{i j}[\phi] \phi_{i}^{*} C_{j}+\frac{1}{2} f^{i j}[\phi] A_{i} \wedge A_{j} .
\end{aligned}
$$

The $f^{i j}[\phi]$ are antisymmetric and to receive a consistent interaction they have to verify

$$
\sum_{c y c l(i j k)} \frac{\delta f^{i j}[\phi]}{\delta \phi^{l}} f^{k l}[\phi]=0,
$$

which is a generalized JACOBI identity. Since this condition is fulfilled there are no obstructions in the construction and the second order deformation can be chosen to be zero. This yields for the deformed solution of the master equation

$$
\begin{aligned}
S_{B V}=\stackrel{(0)}{S}+\stackrel{(1)}{S}=\int_{\Sigma_{g}}[ & A_{i} \wedge \mathrm{d} \phi^{i}-\frac{\delta f^{i j}[\phi]}{\delta \phi^{k}} A^{* k} \wedge A_{i} C_{j}+A^{* i} \wedge \mathrm{d} C_{i}-f^{i j}[\phi] \phi_{i}^{*} C_{j} \\
& \left.+\frac{1}{2} f^{i j}[\phi] A_{i} \wedge A_{j}+\frac{\delta f^{i j}[\phi]}{\delta \phi^{k}} C^{* k} C_{i} C_{j}-\frac{1}{4} \frac{\delta^{2} f^{i j}[\phi]}{\delta \phi^{k} \delta \phi^{l}} A^{* k} \wedge A^{* l} C_{i} C_{j}\right] .
\end{aligned}
$$

From this extended action one can read off the classical action including an interaction term quadratic in the gauge fields $A_{i}$

$$
S_{0}=\stackrel{(0)}{S_{0}}+\stackrel{(1)}{S_{0}}=\int_{\Sigma_{g}}\left[A_{i} \wedge \mathrm{d} \phi^{i}+\frac{1}{2} f^{i j}[\phi] A_{i} \wedge A_{j}\right]
$$

and the deformed gauge symmetries are

$$
\begin{gathered}
\delta_{\epsilon} \phi^{i}=f^{j i}[\phi] \epsilon_{j}, \\
\delta_{\epsilon} A_{i}=\mathrm{d} \epsilon_{i}-f_{, i}^{k l}[\phi] A_{k} \wedge A_{l} .
\end{gathered}
$$

Note, the gauge algebra is only closed on shell, which reflects the non-linearity of the gauge algebra. 


\subsection{The Poisson-Sigma Model}

The Poisson-sigma model is a two-dimensional gauge theory based on a POISsON algebra, i.e. the target manifold of the theory is a Poisson manifold $(N, P)$, that is a smooth manifold $N$ equipped with a Poisson structure $P \in \Lambda^{2} T N$. In local coordinates $X^{i}$ on $N$ the Poisson structure is given by

$$
P=\frac{1}{2} P^{i j}(X) \partial_{i} \wedge \partial_{j}
$$

and $P^{i j}(X)$ has to satisfy the Schouten-NiJenhuis constraint

$$
P^{i[j}(X) P_{, i}^{k l]}(X)=0,
$$

where $[j k l]$ stands for a cyclic sum. That is exactly the consistency condition (2.22) for the structure functions of the deformed two dimensional BF theory. Thus the PoIsson-sigma model is a realization of the deformed Abelian BF theory.

It is possible to extend the action in such a way that the symmetries are unchanged. The POISSON structure induces a map $T^{\star} N \rightarrow T N$ which is not surjective (like in the symplectic case). However, due to the JACOBI identity the image of this map forms an involutive distribution of vector fields. Further the associated characteristic distribution is completely integrable and the POISSON structure induces a symplectic structure $\Omega_{L}$ on the leaves L. It is a fact that a PoIsson manifold is the disjoint union of its symplectic leaves. The splitting theorem of WEINSTEIN states that for regular POISSON manifolds there exist so-called Casimir-Darboux coordinates locally. The Poisson manifolds under consideration in the following sections are isomorphic to $\mathbb{R}^{n}$. For $P$ degenerate there are non vanishing functions $f$ on $N$ whose Hamiltonian vector fields $X_{f} \equiv f_{, i} P^{i j} \partial_{j}$ vanish, the Casimir functions. Then $C^{I}(X)=$ const. $=C^{I}\left(X_{0}\right)$ defines a level surface through $X_{0}$ whose connected components may be identified with the symplectic leaf $L$. This yields natural coordinates $\left\{X^{I}, X^{\alpha}\right\}$ on $(N, P)$, where $\left\{X^{I}\right\}$ is a whole set of CAsimir functions while $X^{\alpha}$ stands for the Darboux coordinates on the leaf $L$ with $P^{I J}=P^{I \alpha}=0$ and $P^{\alpha \beta}=\left(\Omega^{-1}\right)^{\alpha \beta}$. These coordinates will be quite useful in calculating the partition function of the model. The invariant nature of

the CASIMIR functions enables one to add it to the action but the topological invariance of the theory is broken due to the volume form $\mu$ of the world sheet $\Sigma_{g}$. The action and the symmetries are then

$$
\begin{gathered}
S[X, A]=\int_{\Sigma_{g}}\left[A_{i} \wedge \mathrm{d} X^{i}+\frac{1}{2} P^{i j}[X] A_{i} \wedge A_{j}+\mu C[X]\right], \\
\delta_{\epsilon} X^{i}=f^{j i}[X] \epsilon_{j} \quad \delta_{\epsilon} A_{i}=-\mathrm{d} \epsilon_{i}+P_{, i}^{k l}[X] A_{k} \wedge A_{l}=\mathcal{D} \epsilon_{i},
\end{gathered}
$$

it is the action of the Poisson-sigma model introduced in [13].

An interesting aspect of this model is that it associates to certain POISSON structures on a finite-dimensional manifold two-dimensional field theories. First consider the case in which the Poisson structure $P$ gives rise to a symplectic 2 -form on $\mathrm{N}$, i.e. it has an inverse $\Omega$. Then it is possible to eliminate the gauge fields $A_{i}$ by means of the equations of motion and the resulting action is

$$
S_{\text {top }}=\int_{\Sigma_{g}} \Omega_{i j} \mathrm{~d} X^{i} \wedge \mathrm{d} X^{j}
$$


which evidently is the action of Witten's topological sigma Model.

Secondly choose a linear PoISSON structure $P^{i j}=c_{k}^{i j} X^{k}$ on $N=\mathbb{R}^{3}$. The coefficients $c_{k}^{i j}$ then define a LIE algebra on the dual space which in turn can be identified with $N$. Now there are two different CASIMIR functions. The trivial one $C=0$ leads back to the two-dimensional BF theory, although now in the non Abelian case. On the other hand if one chooses now the quadratic CASIMIR function $C=X^{i} X^{i}$ one ends with

$$
S_{Y M}=\int_{\Sigma_{g}} F^{i} \wedge * F_{i}
$$

the two-dimensional Yang-Mills theory. The PoIsson-sigma model also covers the gauged Wess-Zumino-WitTen model and a theory of gravitation [13.

\section{Quantization of the Poisson-sigma Model}

In this section the quantum theory of the PoISSON-sigma model is under consideration. For the Dirac quantization I refer to [13].

\subsection{The Perturbative Series}

This first part is concerned with the perturbation expansion of the path integral of the topological PoIsson-sigma model which yields the KontseviCH quantization formula 顿. It turns out as the correlation function of two functions with support on the boundary of the twodimensional disc, which is the topology of the world sheet

$$
(f \star g)(x)=\int_{X(\infty)=x}[D X][D A] f(X(1)) g(X(0)) \exp \left(\frac{\mathrm{i}}{\hbar} S[X, A]\right),
$$

where $(0,1, \infty)$ are 3 arbitrary, cyclic ordered points on the boundary of the disc. The boundary conditions for all fields are discussed in detail in [4].

In [5] it was shown that the BATALIN-VILKOVISKY action can be obtained by the so-called AKSZ- formalism [1], a geometrical construction for the extended action without a classical action as a starting point. The crucial resulting point is one gets a set of generalized maps, the superfields

$$
\begin{gathered}
\tilde{X}^{i}=X^{i}+\theta^{\mu} A_{\mu}^{* i}-\frac{1}{2} \theta^{\mu} \theta^{\nu} C_{\mu \nu}^{* i}, \\
\tilde{A}_{i}=C_{i}+\theta^{\mu} A_{i}+\frac{1}{2} \theta^{\mu} \theta^{\nu} X_{i \mu \nu}^{*},
\end{gathered}
$$

where $\theta$ are fermionic coordinates of a corresponding super manifold. The action in this extended super space can be formulated as

$$
S_{B V}=\iint_{D^{2}} \mathrm{~d}^{2} \theta\left[\tilde{A}_{i} \mathcal{D} \tilde{X}^{i}+\frac{1}{2} P^{i j}[\tilde{X}] \tilde{A}_{i} \tilde{A}_{j}\right] .
$$


The integration over the fermionic coordinates is in the BERENZIN sense and $\mathcal{D}=\theta^{\mu} \partial / \partial u^{\mu}$. Note that in this formalism the formal form of the action is similar to the original classical one of the PoISSON-sigma model but now the ghosts and the antifields are included in the superfields, in fact after integrating out the fermionic coordinates $\theta$ it is the extended action of the BATALIN-VILKOVISKY formalism (2.23).

Sill in this approach one needs a gauge fixing procedure since the extended action possesses gauge invariances. In the BATALIN-VILKOVISKI formalism the main idea is to introduce a gauge fermion, a functional of the fields only and with ghost number -1 . The integral then is taken over the Lagrangian sub manifold $\Sigma_{\Psi}$ defined by $\Phi_{A}^{*}=\partial \Psi / \partial \Phi^{A}$. The problem is to find a functional $\Psi$ making the integral well-defined. To construct a gauge fermion of ghost number -1 one must introduce additional fields. The simplest choice is a trivial pair $\bar{C}_{i}, \bar{\pi}_{i}$, being LAGRANGE multiplier fields $\bar{\pi}_{i}$ having ghost number 0 and the FADDEEv-Popov antighosts $\bar{C}_{i}$ with ghost number -1 plus the associated antifields. The corresponding action of these fields is $-\int \bar{\pi}_{i} \bar{C}^{* i}$. For calculating the perturbation expansion the natural choice of a gauge is the FEYNMAN gauge $\mathrm{d} \star A_{i}=0$ resulting from the gauge fermion $\Psi=-\int \mathrm{d} \bar{C}^{i} \star A_{i}$ Using this gauge fermion the gauged fixed action takes the form

$$
\begin{aligned}
S_{g f}=\int_{D^{2}}\left[A_{i} \wedge \mathrm{d} X^{i}+\frac{1}{2} P^{i j}[X] A_{i} \wedge A_{j}-\star \mathrm{d} \bar{C}^{i} \wedge\left(\mathrm{d} C_{i}+P_{, i}^{k l}[X] A_{k} C_{l}\right)\right. \\
\left.-\frac{1}{4} \star \mathrm{d} \bar{C}^{i} \wedge \star \mathrm{d} \bar{C}^{j} P^{k l}{ }_{, i j}[X] C_{k} C_{l}-\pi^{i} \mathrm{~d} \star A_{i}\right] .
\end{aligned}
$$

The perturbation expansion is calculated by

$$
\int_{\Sigma_{\psi}}\left[D \Phi_{A}^{*}\right]\left[D \Phi^{A}\right] \exp \left(\frac{\mathrm{i}}{\hbar} S_{g f}\right) \mathcal{O}=\sum_{n=0}^{\infty} \int_{\Sigma_{\psi}}\left[D \Phi_{A}^{*}\right]\left[D \Phi^{A}\right] \exp \left(\frac{\mathrm{i}}{\hbar} S_{g f}^{0}\right)\left(S_{g f}^{1}\right)^{n} \mathcal{O},
$$

where $\mathcal{O}$ denotes the considered observable. The splitting of the action into a kinetic $S_{g f}^{0}$ and a potential $S_{g f}^{1}$ part can be understood as follows. The expansion in powers of $\hbar$ is around the classical solution $X(u)=x, A(u)=0$ and one perturb now the fields $X$ by a fluctuation field $\xi$, e.g. $X(u)=x+\xi(u)$. The kinetic part of the action is then

$$
S_{g f}^{0}=\int_{D^{2}}\left[A_{i} \wedge\left(\mathrm{d} \xi^{i}+\star \mathrm{d} \lambda^{i}\right)+C_{i} \mathrm{~d} \star \mathrm{d} \bar{C}^{i}\right] .
$$

The resulting propagator is expressed most easily in the superfield formalism

$$
\left\langle\tilde{\xi}^{k}(w, \zeta) \tilde{A}_{j}(z, \theta)\right\rangle=\frac{i \hbar}{2 \pi} \delta_{j}^{k} \mathcal{D} \phi(z, w)
$$

with $\phi(z, w)=\frac{1}{2 \mathrm{i}} \ln \frac{(z-w)(z-\bar{w})}{(\bar{z}-\bar{w})(\bar{z}-w)}$ and $z, w$ are denoting two points on the disc.

The perturbation series is then obtained by expanding the potential $S_{g f}^{1}$ in powers of the corresponding superfield of the fluctuation $\tilde{\xi}=\xi+\theta^{\mu} A_{\mu}^{*}$

$$
S_{g f}^{1}=\frac{1}{2} \int_{D^{2}} \int d^{2} \theta \sum_{k=0}^{\infty} \frac{1}{k !} \partial_{j_{1}} \cdots \partial_{j_{k}} P^{i j}(X) \tilde{\xi}^{j_{1}} \cdots \tilde{\xi}^{j_{k}} \tilde{A}_{i} \tilde{A}_{j}
$$


Now expanding the functions $f(\tilde{X}(1))$ and $g(\tilde{X}(0))$ in powers of $\tilde{\xi}$ yields the expansion in FEYNMAN diagrams labeled by $\Gamma_{n}$, where $\mathrm{n}$ is the number of vertices. All the appearing integrals are absolutely convergent, except those containing tadpole diagrams, a diagram with one edge connecting a vertex to itself, such that one has to take care about renormalization. After an usual point-splitting regularization one has to add a resulting counter term which does not spoil the classical master equation.

After these considerations one is in a position to calculate the expansion series with the help of the WICK theorem for GAUssian integrals. The resulting correlation function is then

$$
(f \star g)(x)=f g+\sum_{n=1}^{\infty}\left(\frac{\mathrm{i} \hbar}{2}\right)^{n} \sum_{\Gamma_{n}} w_{\Gamma_{n}} \mathcal{D}_{\Gamma_{n}}(f \otimes g),
$$

where $\mathcal{D}_{\Gamma_{n}}$ denotes the resulting bidifferential operator for the diagram $\Gamma_{n}$ coming from the POISSON structure on the target manifold and $w_{\Gamma_{n}} \approx \int \wedge_{j=1}^{n} \mathrm{~d} \phi\left(u_{j}, u_{v_{1}(j)}\right) \wedge \mathrm{d} \phi\left(u_{j}, u_{v_{2}(j)}\right)$ are the so-called weights which are descended from the propagator. For more details on the calculation, in particular the propagator and the boundary conditions for the fields, take a look at 泪. Note the resulting correlation function is exactly the expression for the product in the deformed algebra of functions on an POISSON manifold invented by Kontsevich [12].

\subsection{Non-perturbative Aspects}

From now on the world sheet is a closed two-dimensional manifold $M$ with genus $g$. Important simplifications occur when one writes the action in CASIMIR-DARBOUX coordinates $\left\{X^{I}, X^{\alpha}\right\}$. The BATALin-VILKOviskY action is then

$$
\begin{aligned}
S_{B V}=\int_{M}\left[A_{I} \wedge \mathrm{d} X^{I}+A_{\alpha} \wedge \mathrm{d} X^{\alpha}+\right. & \frac{1}{2} P^{\alpha \beta}\left[X^{I}\right] A_{\alpha} \wedge A_{\beta}+\mu C\left[X^{I}\right] \\
& \left.+A^{* I} \wedge \mathrm{d} C_{I}+A^{* \alpha} \wedge \mathrm{d} C_{\alpha}+X_{\alpha}^{*} P^{\beta \alpha}\left[X^{I}\right] C_{\beta}\right] .
\end{aligned}
$$

As one can read of the extended action the gauge freedom of the maps $X^{i}: M \rightarrow N$ is reduced to the freedom of the maps $X^{\alpha}: M \rightarrow L$ where $L$ is a symplectic leaf of the PoIsson manifold $N$. The gauge transformations reduce to $\delta_{\epsilon} X^{\alpha}=\left(\Omega^{-1}\right)^{\alpha \beta} \epsilon_{\beta}$ and $\delta_{\epsilon} X^{I}=0$. Hence, after gauge fixing we need to consider only the homotopy classes $\left[X^{\alpha}\right]$. In CAsimir-Darboux coordinates the gauge fermion can be chosen to be

$$
\Psi=\int_{M}\left[\bar{C}^{I} \chi_{I}\left(A_{I}\right)+\bar{C}^{\alpha} \chi_{\alpha}\left(X^{\alpha}\right)\right] .
$$

The gauged fixed action in CASIMIR-DARBOux coordinates is given by

$$
\begin{aligned}
S_{g f}=\int_{M}\left[A_{I}\right. & \wedge \mathrm{d} X^{I}+A_{\alpha} \wedge \mathrm{d} X^{\alpha}+\frac{1}{2} P^{\alpha \beta} A_{\alpha} \wedge A_{\beta}+\mu C\left(X^{I}\right) \\
& \left.+\bar{C}^{J} \frac{\partial \chi_{I}\left(A_{J}\right)}{\partial A_{J}} \wedge \mathrm{d} C_{I}+\bar{C}^{\alpha} \frac{\partial \chi_{\beta}\left(X_{\alpha}\right)}{\partial X_{\alpha}} P^{\gamma \beta} C_{\gamma}-\bar{\pi}^{I} \chi_{I}\left(A_{I}\right)-\bar{\pi}^{\alpha} \chi_{\alpha}\left(X^{\alpha}\right)\right] .
\end{aligned}
$$

This action can now be used to perform the path integral quantization

$$
Z=\int_{\Sigma_{\Psi}}\left[D \Phi_{A}^{*}\right]\left[D \Phi_{A}\right] \exp \left(-\frac{\mathrm{i}}{\hbar} S_{g f}\right) .
$$


It is possible to perform all the integrations of the fields [7]. Integrating over the ghost and antighost fields yields the FADDEEV-POPOV determinants. The integrations over the multipliers yields $\delta$-functions which implement the gauge conditions. From now on the integration extends only over the degrees of freedom with respect to the gauge-fixing conditions. The integration over $A_{\alpha}$ is GaUssian. Now choose a gauge condition linear in $A_{I}$, then the FADDEEV-POPOV determinant does not depend on $A_{I}$ anymore and one can integrate over them yielding a $\delta$-function for $\mathrm{d} X^{I}$. When this $\delta$-function is implemented the fields $X^{I}$ become independent of the coordinates of $M$. Hence the CASIMIR functions are constant and these constant modes $X_{0}^{I}$ count the symplectic leaves. The gauge fixing of the fields $X^{\alpha}$ reduces the integral over $X^{\alpha}$ to a sum over the homotopy classes of the maps. The form of the path integral then becomes

$$
\begin{array}{r}
Z=\int_{\Sigma_{\Psi}} \mathrm{d} X_{0}^{I} \sum_{\left[X^{\alpha}\right]} \operatorname{det}\left(\frac{\partial \chi_{\alpha}\left(X^{\alpha}\right)}{\partial X^{\gamma}} P^{\gamma \beta}\left(X_{0}^{I}\right)\right)_{\Omega^{0}(M)} \operatorname{det}^{-1 / 2}\left(P^{\alpha \beta}\left(X_{0}^{I}\right)\right)_{\Omega^{1}(M)} \\
\quad \times \exp \left(-\frac{\mathrm{i}}{\hbar} \int_{M} \Omega_{\alpha \beta} \mathrm{d} X^{\alpha} \wedge \mathrm{d} X^{\beta}\right) \exp \left(-\frac{1}{\hbar} A_{M} C\left(X_{0}^{I}\right)\right),
\end{array}
$$

where the subscript $\Omega^{k}(M)$ indicates that the determinant results from an integration over $\mathrm{k}$-forms and $A_{M}$ denotes the surface area of $M$. All the functional integrations have been performed and one has arrived at an almost closed expression for the partition function.

Again consider the case in which the manifold $N=\mathbb{R}^{3}$ and the PoIsson structure is linear such that the coefficients give rise to a LIE algebra structure on the dual space $\mathcal{G}=N^{\star}$. The nontrivial CASIMIR function is again the quadratic one, such that the symplectic leaves are two dimensional spheres $\mathbb{S}^{2}$, characterized, in the CASIMIR-DARBOux coordinates, by their radius $X_{0}^{I}$. The symplectic leaves of a linear PoISSON structure are the co-adjoint orbits of the corresponding compact, connected LIE group with LIE algebra $\mathcal{G}$. Because the LIE algebra has three dimensions the LIE group is $\mathrm{SU}(2)$. By a theorem of KIRILLOV [11] these orbits can in turn be identified with the irreducible unitary representations of G.

Taking into account these simplifications the path integral can be evaluated further. Due to the Hopf theorem the homotopy classes of the maps $X^{\alpha}: M \rightarrow \mathbb{S}^{2}$ are determined by their degree $n$. This yields

$$
\int_{M} \Omega_{\alpha \beta} \mathrm{d} X^{\alpha} \wedge \mathrm{d} X^{\beta}=n \int_{\mathbb{S}^{2}} \Omega_{L}\left(X_{0}^{I}\right),
$$

where $\Omega_{L}\left(X_{0}^{I}\right)$ is the symplectic form on the symplectic leaf $\mathrm{L}$ respectively $\mathbb{S}^{2}$. The sum over the degree $n$ yields a periodic $\delta$-function, which says that the symplectic leaves must be integral. By the identification of the leaves with the co-adjoint orbits, they must also be integral which reduces the number of the co-adjoint orbits to a countable set $\mathcal{O}(\Omega)$. Choose now the unitary gauge $\chi^{\alpha}(X)=X^{\alpha}$ and the two determinants have the same form. Due to the Hodge decomposition they are characterized by harmonic forms. Now one has to count the linear independent forms, which are characterized by the dimension of the corresponding homology group, the BeTTI numbers. These yields for the power of the combined determinant the EuLER characteristic $\chi(M)$. Indeed, this is a similar argument used in [3]. The determinant corresponds to the symplectic volume of the symplectic leaf $L_{X_{0}^{I}}$. Now one can 
perform the integration over the constant modes $X_{0}^{I}$. The partition function of the linear PoIsson-sigma model then takes the form

$$
Z=\sum_{\mathcal{O}(\Omega)} \operatorname{Vol}(\Omega)^{\chi(M)} \exp \left(-\frac{1}{\hbar} A_{M} C(\Omega)\right)
$$

As already pointed out it is possible to identify a linear PoIsson manifold with its dual space, the LIE algebra. This duality leads to the similarity of the partition function of the linear Poisson-sigma model (3.17) and the one of the YANG-MiLls theory. The main tool is the so-called symmetrization map [11] which maps in this case the quadratic CASIMIR $C(\Omega)$ which characterized the co-adjoint orbits into the CAsimiR $C(\lambda)$ of the corresponding representation of the LIE group. This leads to the identification of the integral orbits with the irreducible unitary representations of the LIE group. The symplectic volume of the co-adjoint orbit equals by a special case of the character formula of KIRILLOV [11 the dimension of the corresponding representation. So the partition can be written as

$$
Z=\sum_{\lambda} \mathrm{d}(\lambda)^{\chi(M)} \exp \left(-\frac{1}{\hbar} A_{M} C(\lambda)\right),
$$

where $\lambda$ denotes the representation while $d(\lambda)$ stands for its dimension. This is exactly the partition function for the YANG-MILLS theory on closed two-dimensional manifolds [3].

\section{Concluding Remarks}

In this review I presented some interesting aspects of the PoISSON-sigma model discovered in the last years. From the mathematical point of view the most interesting fact is the connection with the deformation quantization of PoIsson manifolds [4]. On the other hand for physicist the important aspect is of course the unified framework for different topological and semi-topological field theories [13] based on the POISSON structure on the target manifold. In the special case of a linear POISSON structure this property could be established also on the quantum level by performing the path integral quantization, i.e. it was shown that the partition function of the linear POISSON-sigma model is in some sense the dual of the one of the YANG-MiLls theory [7].

An interesting step further a whole quantization of the PoIsson-sigma model would be to calculate the partition function on a base manifold with boundaries, in particular on the disc at least for a linear PoISSON structure [8]. The required boundary conditions for the fields must lead to a kind of character formula like the one by KIRILLOV [11] in turn to identify the result with the one for the YANG-MiLls theory where the boundary condition leads to the character of the representation. The hope of this considerations is that it would shed some light on the non-perturbative nature of the deformation quantization.

Acknowledgment This work was supported by the Deutsche Forschungsmeinschaft in connection with the Graduate College for Elementary Particle Physics in Dortmund which gave me the possibility to take part at the 3rd International Conference on Geometry, Integrability and Quantization held in Varna, Bulgaria, June 14-23 2001. 


\section{References}

[1] Alexandrov M., Kontsevich M., Schwarz A., Zaboronsky O., The Geometry of the Master Equation and Topological Quantum Field Theory, Int. J. Mod. Phys. A 12 (1997), $1405-1430$

[2] Barnich G., Henneaux M., Consistent Couplings between Fields with Gauge Freedom and Deformation of the Master Equation, Phys. Lett. B311 (1993), 123-129

[3] Blau M., Thompson G., Lectures on 2d Gauge Theories, e-Print Archive: hepth/9310144

[4] Cattaneo A., Felder G., A Path Integral Approach to the Kontsevich Quantization Formula, Commun. Math. Phys 212 (2000), 591-611

[5] Cattaneo A., Felder G., On the AKSZ formalism of the Poisson-sigma model, Lett. Math. Phys. 56 (2001), 163-170

[6] Gomis J., Paris J., Stuart S., Antibrackets, Antifields and Gauge Theory Quantization, Phys. Rept. 259 (1995), 1-145

[7] Hirshfeld A., Schwarzweller T., Path Integral Quantization of the Poisson-sigma model, Ann. Phys. (Leipzig) 9 (2000) 2, 83-101

[8] Hirshfeld A., Schwarzweller T. (coming soon)

[9] Ikeda N., Two-dimensional Gravity and Non-linear Gauge Theory, Annals Phys. 235 (1994), 435-464

[10] Izawa K.I., On Non-linear Gauge Theory from Deformation Theory Perspective, Prog. Theor. Phys. 103 (2000), 225-228

[11] Kirillov A., Merits and Demerits of the Orbit Method, Bull. Am. Math. Soc., New Ser. 36 No.4 (1999), 433-488

[12] Kontsevich M., Deformation Quantization Of Poisson Manifolds I, e-Print Archive: q-alg/970904

[13] Schaller P., Strobl T., Poisson structure induced (Topological) Field Theories in two Dimensions, Mod. Phys. Lett. A9 (1994), 3129-3136 